\title{
Short-term Time Series Forecasting of Concrete Sewer Pipe Surface Temperature
}

\author{
Karthick Thiyagarajan $^{1}$, Sarath Kodagoda ${ }^{1}$ and Nalika Ulapane ${ }^{2}$
}

\begin{abstract}
Microbial corrosion is considered the main reason for multi-billion dollar sewer asset degradation. Sewer pipe surface temperature is a vital parameter for predicting the micro-biologically induced concrete corrosion. Due to this important measure, a surface temperature sensor suite was recently developed and tested in an aggressive sewer environment. The sensors can fail and they may also put offline during the period of scheduled maintenance. In such situations, time series forecasting of sensor data can be an alternative measure for the operators managing the sewer network. In this regard, this paper focuses on the short-term forecasting of sensor measurements. The evaluation was carried out by forecasting the sensor measurements for different time periods and evaluated with different forecasting models. The ETS model leads to high short-term forecasting accuracy and the ARIMA model leads to high long-term forecasting accuracy. The models were evaluated on real data captured in a Sydney sewer.
\end{abstract}

Keywords - ARIMA model, concrete corrosion, ETS model, forecasting model, sewer pipe, short-term forecasting, surface temperature sensor, temporal, time series.

\section{INTRODUCTION}

Sewerage infrastructure services are essential for transporting wastewater discharges from residential properties, commercial buildings, and industrial areas to domestic sewage treatment plants and systems mainly for treating waterborne wastes and removing pollutants. In Australia, there is more than $140,000 \mathrm{~km}$ of sewer mains that are currently in service. It is widely believed that the sewer pipe system protects the public societies and environments from sewer airborne diseases and bad odors [1]. To safeguard such assets from structural failures, Australian water utilities spend more than AUD 100 million every year in pipe renewals and rehabilitation [2]. A significant proportion of traversable concrete sewer pipes are old in Australia and they are undergoing corrosion because of the microbiological activities that happen on the walls of the pipe surface [3].

Some of the common methods adopted by the water utilities to perform a condition assessment of concrete sewer pipes are visual inspections through closed-circuit television

This work was supported by the Predictive Analytics for Sewer Corrosion Project through in part by the Sydney Water Corporation, in part by the Melbourne Water Corporation, in part by the Water Corporation (Western Australia), and in part by the South Australian Water Corporation. (Corresponding Author: Karthick Thiyagarajan)

${ }^{1}$ Karthick Thiyagarajan and Sarath Kodagoda are with the iPipes Lab, UTS Robotics Institute, Faculty of Engineering and Information Technology, University of Technology Sydney, Sydney, New South Wales, 2007, Australia. Karthick. Thiyagarajan@uts.edu.au, Sarath. Kodagoda@uts.edu.au

${ }^{2}$ Nalika Ulapane is with the School of Electrical and Electronic Engineering, Melbourne School of Engineering, University of Melbourne, Parkville, Victoria, 3052, Australia. Nalika.Ulapane@unimelb.edu.au
(CCTV) based monitoring and drill core analysis [4]. However, the CCTV based assessment can reveal only visual defects on the surface but does not indicate the sub-surface deterioration. Similarly, the drill core analysis may not be an effective method for condition assessment to estimate corrosion. The corroded layers of the concrete can fall off while drilling, which can lead to misinterpretation on the rate of corrosion. Recently, a concept of micro-drilling based sensing technology [5] was reported for estimating the thickness of the corroded concrete layer. This sensor is an invasive type and needs human traversing in sewers for taking measurements like core sampling. Also, there are on-going studies that focus on the feasibility of estimating the depth of acid permeation through electrical resistivity based sensing technology for predicting the corrosion [6]. This reported work is a preliminary investigation through simulation analyses.

The researchers in Australia in collaboration with one of the largest water utility in Australia has developed a predictive analytics model for estimating concrete sewer pipe corrosion [7]. The predictive performance of the model can be improved in areas where there is low confidence in estimating corrosion by supplying concrete surface temperature and moisture conditions as data inputs to the model [8]-[13]. Recently, a robust sensing suite for monitoring the temporal variations of concrete surface temperature conditions was developed [14]. The data inputs from this sensor are crucial for accurate sewer pipe corrosion prediction. Any disruptions to sensor monitoring can have adverse effects on the corrosion monitoring program. For example, sensor monitoring can be halted for a week period due to sensor failure or for a day period due to scheduled sensor suite maintenance. In such circumstances, machine learning algorithms for forecasting sensor data can be crucial for sewer operators to foresee the temperature trends. Moreover, the forecasted sensor data can be an ideal alternative to real sensor measurements during the disrupted monitoring period. Another benefit that can be derived from the forecast data is the detection of anomalies and early sensor failure prediction by comparing the forecast data with the streaming sensor measurements [15], [16]. Hence, the forecasted sensor data is important and it can be fed as surface temperature data input to the predictive analytics model for estimating corrosion. In this context, this paper focuses on conducting performance evaluation on relevant time series models for forecasting the concrete sewer pipe surface temperature sensor measurements. Each model's forecast was statistically evaluated against the sensor data collected from field tests conducted in Sydney, Australia. 
The rest of this paper is organized as follows: Section II briefly reviews the related work. Section III describes the methods used in this paper. Section IV presents the evaluation results with analysis and finally, Section $\mathrm{V}$ concludes the paper by summarizing the key outcomes and briefing the future work.

\section{BRIEF REVIEW ON RELATED WORK}

There are several studies available in the literature on forecasting sensor data. However, limited studies were reported on the models of forecasting parameters that are related to the sewer pipe environment. Zhang et al. conducted experiments to find an efficient approach for forecasting the wastewater flow in sewer pipes by comparing the performance of the Autoregressive Integrated Moving Average (ARIMA) model with the Multilayer Perceptron Neural Network (MLPNN) model [17]. The outcomes of the study indicated that both models can be used for forecasting. However, the performance of the ARIMA model was better when statistically evaluated with the benchmark metrics. Another study developed a forecasting based anomaly detection model for surface moisture sensor monitoring inside the sewer pipe [10]. The study evaluated different time series forecasting models such as the Bagged model, Exponential Smoothing with error, trend and seasonality (ETS) model, and the seasonal ARIMA model combined with Hyndman and Khandakar algorithm [18]. The study outcomes demonstrated that the forecasting performance of the seasonal ARIMA model was better than the forecasts of other models for forecasting one week period. Also, daily forecasts were also made by the seasonal ARIMA model in developing the anomaly detection model. Since the seasonal ARIMA model in that study is combined with Hyndman and Khandakar algorithm, the optimization parameters of the model are chosen automatically. This makes the forecasting model a hybrid type because of the reason that the ARIMA model optimization parameters can control the forecasting model to be either the ARIMA model or Autoregressive (AR) model or Moving Average (MA) model [16]. Similar studies were conducted for the sewer pipe surface temperature sensor, where the seasonal ARIMA model was used for forecasting a day period to develop an early sensor failure prediction model [15]. The temporal forecasting performance of different models such as Facebook's Prophet method, TBATS model, ARIMA model, ETS model, and the Bagged model were evaluated for forecasting sewer air temperature sensor data. The study outcomes indicated that the performance of Facebook's Prophet method was better in terms of accuracy than other forecasting models for forecasting a week period and a daily period [19]. In this paper, we use the surface temperature sensor data to evaluate the forecasting performances of the seasonal ARIMA model combined with Hyndman and Khandakar algorithm, Facebook Prophet method, ETS model, and Bagged model.

\section{METHODS}

\section{A. Field Data Collection}

An infrared radiometer is a non-contact type sensing element of the concrete sewer pipe surface temperature sensor suite. The sensor was deployed inside the sewer by installing the sensing unit near the crown of the pipe. The sensor measurements were collected and stored in a computing platform in the monitoring station, which is constructed outside the pipe. The measurements were collected from the sewer pipe managed by the Sydney Water Corporation at the Thornleigh suburb, Sydney, Australia. For more information on the sensor development and field tests, the readers can refer to [14]. The surface temperature data will be used to evaluate the forecasting models.

\section{B. Time Series Forecasting Models}

Let $T_{M(t)}$ be the temperature sensor data at $t$ instantaneous time. The past hour sensor measurement is represented as $T_{M(t-1)}$.

1) ARIMA Model: The ARIMA model is the most popular and widely used method for forecasting time series data [20]. This model has three components namely autoregressive (AR) component. moving average (MA) and integrated component the The forecast value of the seasonal ARIMA model is given by (1).

$$
\begin{array}{r}
\left(1-\sum_{x=1}^{p} \phi_{x} \beta^{x}\right)\left(1-\sum_{x=1}^{P} \Phi_{x} \beta^{T_{M p}}\right)(\Delta)^{d}\left(\Delta^{T_{M p}}\right)^{D} \widetilde{T_{M(t)}} \\
=\left(1+\sum_{y=1}^{q} \theta_{y} \beta^{y}\right)\left(1+\sum_{y=1}^{Q} \Theta_{y} \beta^{T_{M p}}\right) \varepsilon_{t}
\end{array}
$$

where the AR component order is $p$, MA component order is $q$, differencing level is $d, \phi$ is the AR component weight parameter, $\theta$ is the MA component weight parameter, $\beta$ is the backshift operator, $T_{M p}$ is the seasonal period, seasonal AR component order is $P$, seasonal MA component order is $Q$, differencing parameter is $\Delta$, seasonal differencing level is $D, \Phi$ is the seasonal AR component weight parameter and $\Theta$ is the seasonal MA component weight parameter. The value $p, d, q, P, D$ and $Q$ are chosen automatically through the optimization algorithm [18]. The white noise is $\varepsilon_{t}$ and the mean deviation is $\widetilde{T}_{M(t)}$.

2) ETS Model: The point forecast of the ETS model [21] is given by $Y_{t}$, where the $Y_{t}=Y_{t+1}+Y_{t+2}+\ldots .+Y_{t+n}$ and $n$ is the finite number. The previous value $Y_{t+1}$ and $Y_{t+2}$ are given in (2) and (3) respectively.

$$
Y_{t+1}=\left(L_{t}+B_{t}\right)\left(1+\epsilon_{t+1}\right)
$$

where $L_{t}$ is the series level at $t$ instantaneous time, $B_{t}$ is the slope level at $t$ and $\epsilon$ denotes the error.

$$
Y_{t+2}=\left(L_{t+1}+B_{t+1}\right)\left(1+\epsilon_{t+1}\right)
$$




$$
\begin{aligned}
\left(L_{t+1}+B_{t+1}\right)\left(1+\epsilon_{t+1}\right)= & {\left[\left(L_{t}+B_{t}\right)\left(1+\alpha \epsilon_{t+1}\right)+B_{t}\right.} \\
+ & \left.\left.\beta\left(L_{t}+B_{t}\right) \epsilon_{t+1}\right)\right]\left(1+\epsilon_{t+1}\right)
\end{aligned}
$$

where $\alpha$ and $\beta$ are the smoothing parameters.

3) Prophet Model: The Prophet model is a forecasting method developed by the researchers in Facebook [22]. The Prophet model forecast value $P(t)$ at time $t$ is given by (5):

$$
P_{(t)}=G_{(t)}+S_{(t)}+H_{(t)}+\epsilon_{t}
$$

where $\epsilon_{t}$ is the model error. The term $H_{(t)}$ denotes the holiday effect, where in the application reported in this paper $H_{(t)}=0, G_{(t)}$ is the model's growth trend expressed as in (6) and $S_{(t)}$ is the model's seasonality expressed as in (7).

$$
G_{(t)}=\frac{C}{1+\exp (-K(t-M))}
$$

where $C$ is the carrying capacity, $K$ is the logistic growth rate and the offset parameter is $M$.

$$
S_{(t)}=\sum_{n=1}^{n=N}\left(A_{n} \cos \left(\frac{2 \pi n t}{P}\right)+B_{n} \sin \left(\frac{2 \pi n t}{P}\right)\right)
$$

where $P$ is the forecasting period, where $P=0.5$ when the model forecasts for 12 hours, $P=1$ when the model forecasts for one day and $P=7$ when the model forecasts for one week. $A_{n}$ and $B_{n}$ are the seasonal parameters, $n$ is the seasonal value and the instantaneous time is $t$.

4) Bagged Model: The Bagged model forecasting is described in [23]. We have used the Forecast package in $\mathrm{R}$ Studio for implementing this model.

\section{Performance Indicators}

The error metrics such as Mean Absolute Error (MAE), Root Mean Square Error (RMSE) and Mean Absolute Percentage Deviation (MAPD) were used as the performance indicators to evaluate the forecasting models. The MAE expressed in (8) represents the modulus of the average magnitude difference between the model forecast values and sensor measurements whereas the RMSE expression in (9) indicates the standard deviation of the forecast errors. The MAPD defined in (10) gives the percentage of the absolute average deviation between the forecast and measured data.

$$
\begin{aligned}
M A E & =\frac{1}{S_{D}} \sum_{t=1}^{t=S_{D}}\left|M_{F(t)}-T_{M(t)}\right| \\
R M S E & =\sqrt{\frac{1}{S_{D}} \sum_{t=1}^{t=S_{D}}\left(M_{F(t)}-T_{M(t)}\right)^{2}} \\
M A P D & =\frac{100 \%}{S_{D}} \sum_{t=1}^{t=S_{D}}\left|\frac{M_{F(t)}-T_{M(t)}}{T_{M(t)}}\right|
\end{aligned}
$$

where the terms $M_{F(t)}$ and $T_{M(t)}$ denotes the data of the forecasting model and the temperature sensor data respectively at the instantaneous time $t$. The $S_{D}$ is the size of the forecast and sensor data.

\section{Evaluation Results}

This section presents the evaluation results of the forecasting models. The surface temperature sensor data collected inside the sewer pipe were used for evaluating the forecasting models. The sensor measurements were taken in a periodic interval of one hour. The first sensor measurement of the day was taken at time 00:00 hours and the last measurement was taken at time 23:00 hours. In total, there are 24 measured values per day. The forecasting models such as the ARIMA model, the ETS model, the Prophet model, and the Bagged model were trained by using one-week sensor data. The one-week period is between $4^{\text {th }}$ November 2016 and $10^{\text {th }}$ November 2016. All-together, 168 sensor data samples were used for training the forecast model. The trends of the sensor data used for training is shown in Fig. 1.

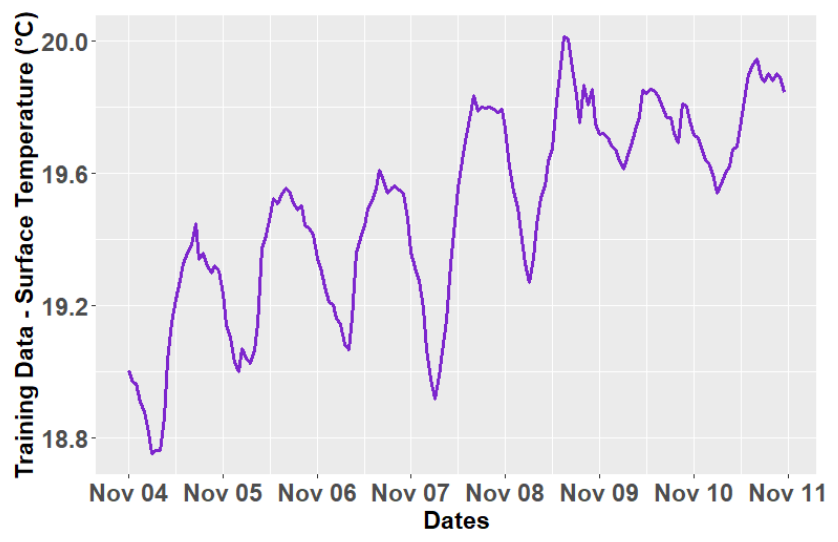

Fig. 1: Training data inputs for the forecasting models.

By training the forecasting models, we forecast the concrete sewer pipe surface temperature sensor measurements for one-week. The forecast week period is between $11^{\text {th }}$ November 2016 and $17^{\text {th }}$ November 2016. Figure 2 shows the forecasted sensor measurement values by each forecasting model along with the actual sensor data collected from the sewer pipe.

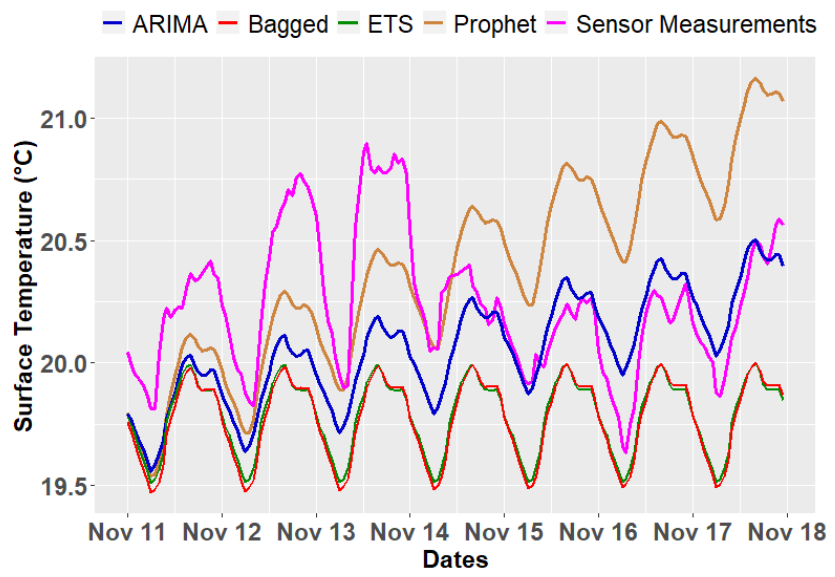

Fig. 2: Temporal forecasts for one week period.

It can be observed from Fig. 2 that the forecasts of the Prophet model and the ARIMA model are having an 
increasing trend, which is similar to the training data trend. However, there is a difference in the increasing trend between the two models. Also, the difference in forecast values between the Prophet model and the ARIMA model is increasing each day. An identical trend can be observed in Fig. 2 for the forecasts of the ETS model and the Bagged model. However, there are slight differences in the forecast values of those two models.

The performance indicators were determined for the forecasts of each forecasting model. Table I tabulates the computed results, where it can be observed that the ARIMA model forecasts have the lowest MAE, RMSE, and MAPD values compared to the Prophet model, the ETS model and, the Bagged model. The Prophet model has the second-lowest values on the performance indicators and the ETS model has the third-lowest values. The Bagged model has the highest values on all the performance indicators, which makes it the least performing model.

TABLE I: Forecasting Evaluation For One Week Period

\begin{tabular}{|c|c|c|c|}
\hline \multirow{2}{*}{ Forecasting Models } & \multicolumn{3}{|c|}{ Performance Indicators } \\
\cline { 2 - 4 } & MAE $\left({ }^{\circ} \mathbf{C}\right)$ & RMSE $\left({ }^{\circ} \mathbf{C}\right)$ & MAPD (\%) \\
\hline ARIMA Model & 0.2648 & 0.3382 & 1.2994 \\
\hline ETS Model & 0.4583 & 0.5006 & 2.2528 \\
\hline Prophet Model & 0.4147 & 0.4669 & 2.0490 \\
\hline Bagged Model & 0.4995 & 0.5372 & 2.4541 \\
\hline
\end{tabular}

From Table I, it can be noticed that the difference in MAE and RMSE between the forecasts of the ARIMA model and the Prophet model is approximately $0.15^{\circ} \mathrm{C}$ and $0.13^{\circ} \mathrm{C}$ respectively whereas the MAE and RMSE difference between the ARIMA model and ETS model forecast is $0.19^{\circ} \mathrm{C}$ and $0.16^{\circ} \mathrm{C}$ respectively, and $0.23^{\circ} \mathrm{C}$ and $0.20^{\circ} \mathrm{C}$ is the MAE and RMSE difference between the Bagged model and the ARIMA model forecast. The maximum difference in MAPD between the forecasts of the ARIMA model and other models is less than $1.16 \%$. This analysis shows that the forecasting performance of the ARIMA model is better than the other models. Therefore, it can be concluded that the ARIMA model is suitable for forecasting one-week concrete sewer pipe surface temperature sensor measurements.

In the previous forecasting evaluation, we forecasted for one week. In the next analysis, we forecast the sensor measurements only for 24 hour period. The first-day forecast was made by training each forecasting model using the sensor data as shown in Fig. 1. Then, the second-day sensor measurements were forecasted by updating the training data with the previous day's actual sensor measurements. Similarly, upcoming days were forecasted by updating the sensor measurements of the previous day. This process goes on till the seventh day. Figure 3 shows the 24 hours forecasts of each model along with the actual sensor data collected from the sewer pipe for one week.

Table II tabulates the computed performance indicators for 24 hour period forecasting, where it can be observed that

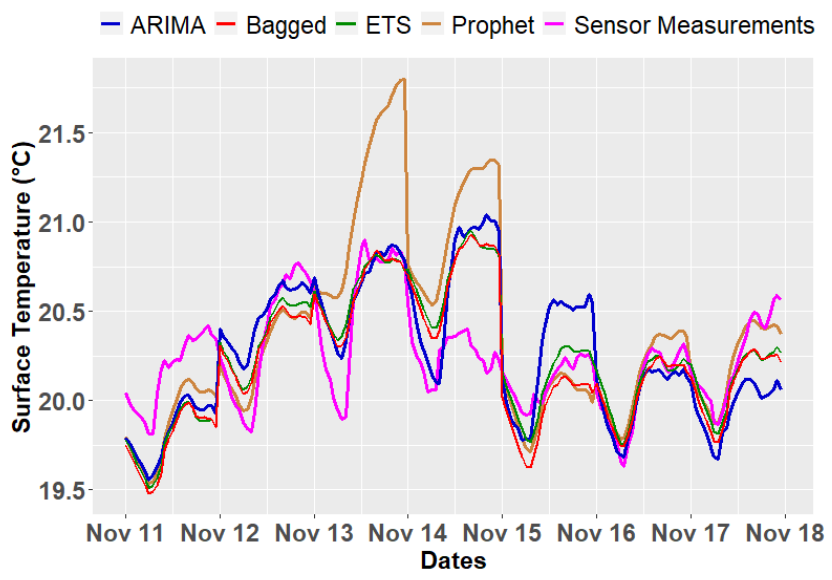

Fig. 3: Temporal forecasts for 24 hour period.

the forecasts of the ETS model have the lowest values on all the performance indicators than the ARIMA model, the Prophet model, and the Bagged model. The Bagged model has the second-lowest values on the performance indicators and the ARIMA model has the third-lowest values. The Prophet model has the highest values on all the performance indicators, which makes it the least performing model for forecasting 24 hour period.

TABLE II: Forecasting Evaluation For 24 Hour Period

\begin{tabular}{|c|c|c|c|}
\hline \multirow{2}{*}{ Forecasting Models } & \multicolumn{3}{|c|}{ Performance Indicators } \\
\cline { 2 - 4 } & MAE $\left({ }^{\circ} \mathbf{C}\right)$ & RMSE $\left({ }^{\circ} \mathbf{C}\right)$ & MAPD (\%) \\
\hline ARIMA Model & 0.2388 & 0.3021 & 1.1812 \\
\hline ETS Model & 0.1967 & 0.2641 & 0.9729 \\
\hline Prophet Model & 0.2897 & 0.4155 & 1.4253 \\
\hline Bagged Model & 0.2125 & 0.2723 & 1.0575 \\
\hline
\end{tabular}

From Table II, it can be noticed that the difference in MAE between the ETS model and other models is less than $0.10^{\circ} \mathrm{C}$. Similarly, the difference in RMSE between the models is less than $0.15^{\circ} \mathrm{C}$. Also, the difference in MAPD between the forecasts of the ETS model and other models is small. Although the difference in performance indicators between the models is very small, the ETS model has performed better forecasting for 24 hour period. Also, it is to be noted that the MAE and RMSE of the ETS model forecast have reduced almost by $50 \%$ from the performance indicator values of one-week forecasts.

In the previous forecasting evaluation, we forecasted for one day period. In our third analysis, we forecast the sensor measurements only for 12 hour period. By using the training data shown in Fig. 1, we forecast for 12 hours. Then, the training model is updated with the actual sensor measurements for forecasting the next 12 hour period. This forecasting process is continued for one week, where Fig. 4 shows the temporal forecasts for each model for 12 hours. It can be observed from Fig. 4 that the forecasts of all the models follow a similar trend as the actual sensor 
measurements

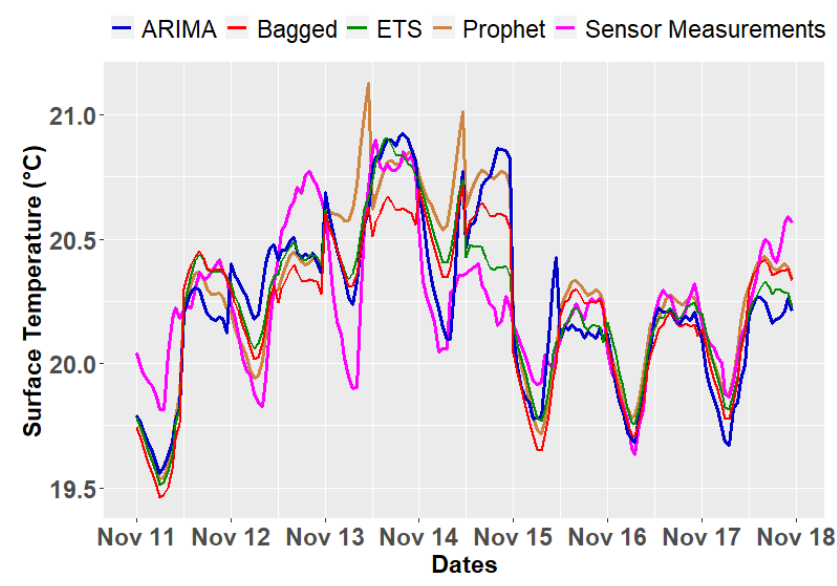

Fig. 4: Temporal forecasts for 12 hour period.

Table III tabulates the computed performance indicators for 12 hour period forecasting, where it can be observed that the ETS model's forecasts have the lowest values on the MAE, RMSE, and MAPD than all other models. Also, it is to be noted that the difference between performance indicators values of each model is minimal. Similar to 24 hour forecasting, the ETS model has high forecasting accuracy followed by the Bagged model, the ARIMA model, and the Prophet model. The MAE and RMSE of the ETS model forecast of 12 hour period have lower values compared to the performance indicator values of 24 hour period forecast. Therefore, the ETS model is also suited for forecasting 12 hour period.

TABLE III: Forecasting Evaluation For 12 Hour Period

\begin{tabular}{|c|c|c|c|}
\hline \multirow{2}{*}{ Forecasting Models } & \multicolumn{3}{|c|}{ Performance Indicators } \\
\cline { 2 - 4 } & MAE $\left({ }^{\circ} \mathbf{C}\right)$ & RMSE $\left.^{\circ}{ }^{\circ} \mathbf{C}\right)$ & MAPD (\%) \\
\hline ARIMA Model & 0.1848 & 0.2343 & 0.9137 \\
\hline ETS Model & 0.1457 & 0.1912 & 0.7295 \\
\hline Prophet Model & 0.1853 & 0.2607 & 0.9162 \\
\hline Bagged Model & 0.1800 & 0.2230 & 0.8811 \\
\hline
\end{tabular}

\section{CONClusions AND Future Work}

This paper focuses on forecasting short-term temporal dynamics of concrete sewer pipe surface temperature sensor measurements. The forecasting performances of the ARIMA model, the Prophet model, the ETS model, and the Bagged model were evaluated by forecasting surface temperature sensor measurements for the periods of one week, 24 hours, and 12 hours. The error metrics such as MAE, RMSE, and MAPD were used as performance indicators of forecasting models. The ARIMA model forecast values for one week have the lowest values on performance indicators compared to other models. This indicates the ARIMA model's better performance and its suitability for forecasting sensor measurements one-week ahead. However, the ETS model has the lowest values on performance indicators for the forecasting period of 24 hours and 12 hours. This shows that the ETS model's performance is better for forecasting 24 hours and 12 hours. Also, the forecasting errors of the ETS model for 12 hours has decreased from one week and 24 hour period forecasting. This demonstrates the improved forecasting performance of the ETS model. Therefore, the ETS model is recommended for forecasting one day and 12 hour period surface temperature sensor measurements. In the future, the proposed ETS forecasting approach will be extended to develop an anomaly detection and early sensor failure estimation algorithm for the concrete sewer pipe surface temperature sensor suite.

\section{ACKNOWLEDGMENT}

This work was supported by the Predictive Analytics for Sewer Corrosion Project through in part by the Sydney Water Corporation, in part by the Melbourne Water Corporation, in part by the Water Corporation (WA), and in part by the South Australian Water Corporation. The research participants are Data61 - Commonwealth Scientific and Industrial Research Organization (CSIRO), University of Technology Sydney (UTS), and The University of Newcastle (UoN). The authors would like to acknowledge Dammika Vitanage, Gino Iori, Craig Earl, and his team from the Sydney Water Corporation for their contribution to sensor suite development and sensor tests inside the sewer pipe managed by Sydney Water Corporation.

\section{REFERENCES}

[1] I. Pikaar, K. R. Sharma, S. Hu, W. Gernjak, J. Keller, and Z. Yuan, "Reducing sewer corrosion through integrated urban water management," Science, vol. 345, no. 6198, pp. 812-814, 2014.

[2] P. Wells and R. E. Melchers, "Microbial Corrosion of Sewer Pipe in Australia Initial Field Results," in 18th International Corrosion Congress Proceedings November, 2011.

[3] G. Jiang, J. Keller, and P. L. Bond, "Determining the long-term effects of $\mathrm{H} 2 \mathrm{~S}$ concentration, relative humidity and air temperature on concrete sewer corrosion," Water research, vol. 65, pp. 157-169, 2014.

[4] N. Stanić, C. d. Haan, M. Tirion, J. Langeveld, and F. H. L. R. Clemens, "Comparison of core sampling and visual inspection for assessment of concrete sewer pipe condition," vol. 67, no. 11, pp. 2458-2466, 2013

[5] N. Giovanangeli, L. Piyathilaka, S. Kodagoda, K. Thiyagarajan, S. Barclay, and D. Vitanage, "Design and development of drillresistance sensor technology for accurately measuring microbiologically corroded concrete depths," in Proceedings of the 36th International Symposium on Automation and Robotics in Construction. IAARC, 2019, pp. 735-742.

[6] K. Thiyagarajan, P. Acharya, L. Piyathilaka, and S. Kodagoda, "Numerical Modeling of the Effects of Electrode Spacing and Multilayered Concrete Resistivity on the Apparent Resistivity Measured Using Wenner Method." TechRxiv., 2020.

[7] B. Li, X. Fan, J. Zhang, Y. Wang, F. Chen, S. Kodagoda, T. Wells, L. Vorreiter, D. Vitanage, G. Iori, D. Cunningham, and T. Chen, "Predictive Analytics Toolkit for H2S Estimation and Sewer Corrosion," in OZWater. Sydney: Australian Water Association, 2017.

[8] K. Thiyagarajan, "Robust Sensor Technologies Combined with Smart Predictive Analytics for Hostile Sewer Infrastructures," Ph.D. dissertation, University of Technology Sydney, 2018.

[9] K. Thiyagarajan, S. Kodagoda, and J. K. Alvarez, "An instrumentation system for smart monitoring of surface temperature," in 2016 14th International Conference on Control, Automation, Robotics and Vision (ICARCV), 2016, pp. 1-6. 
[10] K. Thiyagarajan, S. Kodagoda, R. Ranasinghe, G. Iori, and D. Vitanage, "Robust Sensor Suite Combined with Predictive Analytics Enabled Anomaly Detection Model for Smart Monitoring of Concrete Sewer Pipe Surface Moisture Conditions," IEEE Sensors Journal, vol. 20 , no. 15 , pp. 8232-8243, 2020.

[11] K. Thiyagarajan, S. Kodagoda, and N. Ulapane, "Data-driven machine learning approach for predicting volumetric moisture content of concrete using resistance sensor measurements," in 2016 IEEE 11th Conference on Industrial Electronics and Applications (ICIEA), 2016, pp. $1288-1293$.

[12] K. Thiyagarajan and S. Kodagoda, "Analytical Model and Data-driven Approach for Concrete Moisture Prediction," in 33rd International Symposium on Automation and Robotics in Construction (ISARC 2016). Auburn: IAARC, 2016, pp. 298-306.

[13] K. Thiyagarajan, S. Kodagoda, L. V. Nguyen, and S. Wickramanayake, "Gaussian Markov Random Fields for Localizing Reinforcing Bars in Concrete Infrastructure," in 2018 Proceedings of the 35th International Symposium on Automation and Robotics in Construction. Berlin: IAARC, 2018, pp. 1052-1058.

[14] K. Thiyagarajan, S. Kodagoda, R. Ranasinghe, D. Vitanage, and G. Iori, "Robust sensing suite for measuring temporal dynamics of surface temperature in sewers," Scientific Reports, vol. 8, no. 1 , p. 16020,2018 . [Online]. Available: http://www.nature.com/articles/s41598-018-34121-3

[15] K. Thiyagarajan, S. Kodagoda, L. V. Nguyen, and R. Ranasinghe, "Sensor Failure Detection and Faulty Data Accommodation Approach for Instrumented Wastewater Infrastructures," IEEE Access, vol. 6, pp. 56562-56574, 2018.

[16] K. Thiyagarajan, S. Kodagoda, and L. Nguyen, "Predictive Analytics for Detecting Sensor Failure Using Autoregressive Integrated Moving Average Model," in 12th IEEE Conference on Industrial Electronics and Applications. Siem Reap: IEEE, 2017, pp. 1923-1928.

[17] Q. Zhang, Z. Li, S. Snowling, A. Siam, and W. El-Dakhakhni, "Predictive models for wastewater flow forecasting based on time series analysis and artificial neural network," Water Science and Technology, vol. 80, no. 2, 2019 .

[18] R. J. Hyndman and Y. Khandakar, "Automatic time series forecasting:
The forecast package for R," Journal Of Statistical Software, 2008.

[19] K. Thiyagarajan, S. Kodagoda, N. Ulapane, and M. Prasad, "A Temporal Forecasting Driven Approach Using Facebook's Prophet Method for Anomaly Detection in Sewer Air Temperature Sensor System.” TechRxiv., 2020.

[20] G. E. P. Box, G. M. Jenkins, and G. C. Reinsel, "Time Series Analysis: Forecasting and Control," Journal of Time Series Analysis, 2013.

[21] R. Hyndman, A. Koehler, J. Ord, and R. Snyder, Forecasting with Exponential Smoothing The State Space Approach. Berlin: SpringerVerlag, 2008.

[22] S. J. Taylor and B. Letham, "Forecasting at Scale," American Statistician, vol. 72, no. 1, pp. 37-45, 2018

[23] C. Bergmeir, R. J. Hyndman, and J. M. Benítez, "Bagging exponential smoothing methods using STL decomposition and Box-Cox transformation," International Journal of Forecasting, vol. 32, no. 2, pp. 303312, 2016. 